\title{
Early assessment of 5-fluorouracil and bevacizumab induced cardiotoxicity by multimodal imaging techniques
}

\author{
Xuchang Chen ${ }^{1}$, Weimin $\mathrm{Han}^{2}$, Huimin $\mathrm{Wang}^{3}$, Yueming $\mathrm{Wu}^{1}$, and maolong $\mathrm{SU}^{3}$ \\ ${ }^{1}$ Fujian Medical University \\ ${ }^{2}$ School of Medicine, Xiamen University \\ ${ }^{3}$ Department of Ultrasonography, Xiamen Cardiovascular Hospital
}

June 4, 2020

\begin{abstract}
Cardiotoxicity, including acute and late-onset cardiotoxicity, was a well-known adverse effect of many types of antitumor agents. Early identification of patients with cardiotoxicity was important to ensure prompt treatment and minimize toxic effects. This study compared the potential ability of multinomial technique in early detection, prediction and combined diagnosis of chemotherapy induced cardiotoxicity. We constructed a chemotherapy induced cardiotoxicity model through intraperitoneal injection bevacizumab and 5-fluorouracil in Balb/c mice. These mice were administered with electrocardiogram, conventional echocardiography, speckle tracking echocardiography, PET-CT, detection of B-type natriuretic peptide, myocardial pathology. Compared with other cardiac diagnose techniques, speckle tracking echocardiography and 18F-FDG myocardial imaging can detect myocardial injury in the early stage. The global longitudinal strain may provide a noninvasive and accurate method for the early detection of cardiotoxicity. The long axis longitudinal strain of left ventricular first decreased in the apical segment of left ventricle. The long axis radial strain of left ventricular first decreased in the posterior segment of left ventricle. The short axis radial strain and circumferential strain of left ventricular first decreased in the anterior wall of left ventricle.
\end{abstract}

Early assessment of 5 -fluorouracil and bevacizumab induced cardiotoxicity by multimodal imaging techniques

Chen Xuchang ${ }^{1}$, Han Weimin ${ }^{3}$, Wang Huimin ${ }^{2}$, Wu Yueming ${ }^{1}$, Su Maolong ${ }^{2}$

${ }^{1}$ The School of Clinical Medicine, Fujian Medical University, Fuzhou 350100, China; ${ }^{2}$ Department of Ultrasonography, Xiamen Cardiovascular Hospital, Xiamen University, Xiamen 361009, China; ${ }^{3}$ School of Medicine, Xiamen University, Xiamen 361005, China;

${ }^{2}$ Corresponding author: Su Maolong, Email: sumaolong@xmu.edu.cn

\section{【Abstract】}

Cardiotoxicity, including acute and late-onset cardiotoxicity, was a well-known adverse effect of many types of antitumor agents. Early identification of patients with cardiotoxicity was important to ensure prompt treatment and minimize toxic effects. This study compared the potential ability of multinomial technique in early detection, prediction and combined diagnosis of chemotherapy induced cardiotoxicity. We constructed a chemotherapy induced cardiotoxicity model through intraperitoneal injection bevacizumab and 5 -fluorouracil in Balb/c mice. These mice were administered with electrocardiogram, conventional echocardiography, speckle tracking echocardiography, PET-CT, detection of B-type natriuretic peptide, myocardial pathology. Compared with other cardiac diagnose techniques, speckle tracking echocardiography and ${ }^{18} \mathrm{~F}-$ FDG myocardial imaging can detect myocardial injury in the early stage. The global longitudinal strain may provide a noninvasive and accurate method for the early detection of cardiotoxicity. The long axis 
longitudinal strain of left ventricular first decreased in the apical segment of left ventricle. The long axis radial strain of left ventricular first decreased in the posterior segment of left ventricle. The short axis radial strain and circumferential strain of left ventricular first decreased in the anterior wall of left ventricle.

【Key words】 chemotherapy induced cardiotoxicity; speckle tracking; preclinical mode

Fund program: Xiamen Key Project of Medical and Health Sciences(3502Z20191103);

ORCID:0000-0002-0855-7079

\section{Background}

According to the 2018 Global Cancer Statistics provided by the International Agency for Research on Cancer, the number of new cancer cases were 18.1 million and 9.6 million death cases global in $2018^{\lfloor 1 】}$. Colorectal cancer was one of the most common malignant tumors in the world, accounting for $10 \%$ of all malignant tumors, which ranks the third in the malignant tumors. In China, colorectal cancer was the fourth third leading cause of cancer-associated mortality in men and the third in women ${ }^{[2,3]}$. With the continuous progress and maturity of radiotherapy and chemotherapy technology, the survival rate of patients has improved through the application of anti-tumor drugs alone or combined with radiotherapy. However, with the development of chemotherapy, anthracycline was introduced into the field of tumor therapy in 1960s, people have come to realize the cardiotoxicity caused by chemotherapy for the first time. With the development of medical technology, cardiotoxicity caused by anti-tumor drugs was gradually familiar and valued by experts of various disciplines. It was recognized that chemotherapy induced cardiotoxicity restrict the long-term survival of patients to a certain extent, and increased the global public health burden. In high-risk patients, prevention of heart failure caused by chemotherapy was crucial to their long-term health. This would not only increase their risk of cardiovascular disease and death, but also may limit their ability to receive adequate cancer treatment. Therefore, early detection of chemotherapy induced cardiotoxicity was very important.

Cardiooncology was an emerging field that aimed to optimize cancer treatment options and manage heart disease caused by cancer treatment. In the past decade, cancer therapy has been greatly developed, including many targeted therapies based on tumor genetics and receptor characteristics [4】. However, these were accompanied by a series of cardiovascular complications, including heart failure and left ventricular systolic dysfunction, myocarditis, arrhythmia, coronary artery, pericardial and valvular heart disease. More and more cardiac imaging technology, accompanied by ever-increasing technological developments, was applied to the diagnosis of diseases, such as speckle tracking echocardiography, PET-CT cardiac magnetic resonance and so on. But the best imaging method or biomarker to diagnose chemotherapy induced cardiotoxicity was still controversial.

The 5-fluorouracil and bevacizumab were widely used in the treatment of colorectal cancer ${ }^{5}$ \, but the early monitoring method of the structure and function of heart injury caused by this combination of the drugs was not perfect. This study used different monitoring methods to compare the changes of cardiac structure and function before and after the combination of bevacizumab and 5-fluorouracil injection in mice model. The purpose of this study was to explore a safe and effective detection method with strong clinical operability, which is in order to find an early detection of clinical chemotherapy induced cardiotoxicity.

\section{Methods}

Experimental animals: The healthy male Balb/c mice, purchased from Wu's Animal Center, Fuzhou City, Fujian Province, were free to drink water in separate cages and fed with granular feed. Weigh the mice regularly. The ambient temperature was 2426 , and the humidity was $60 \% 80 \%$. The animals were randomly divided into two groups, blank control group and 5-fluorouracil + bevacizumab group. In the 5- fluorouracil + bevacizumab group, $5 \mathrm{mg} / \mathrm{kg}$ bevacizumab and $30 \mathrm{mg} / \mathrm{kg}$ fluorouracil were injected intraperitoneally once every two day for 14 days. All animal procedures were approved by the Animal Ethics Committee of Fujian Medical University. 
Echocardiography: Echocardiography was performed before injection and on the 4th, 7th and 14th day after injection. The mice were anesthetized with isoflurane inhalation at an induced concentration of $3 \%$. Then, the isoflurane at a maintained concentration of $1 \% 1.5 \%$ was administered to maintain anesthesia, and the target heart rate was maintained at 400500 times per minute. After anesthesia, the mice were lying on the operating platform with a temperature of 40 , and the heart rate was recorded by sticking all four limbs on the electrode piece of the operating platform.

The function of heart was analyzed by echocardiography with Visual Sonic Vevo 2100, and B mode and M mode echocardiography of long axis and short axis of left ventricle were detected by MS550D high frequency ultrasonic probe. And then measure IVS (interventricular septum thickness), LVPW (left ventricular posterior wall thickness), lVID;d (left ventricular end diastolic dimension), lVID;s (left ventricular end systolic dimension), LVEF (left ventricular ejection fraction) and LVFS (left ventricular fraction shortening). Then the apical four-chamber view was used the color doppler mode and spectrum doppler mode to record MVE (early diastolic mitral valve flow velocity), MVA (early diastolic mitral valve flow velocity) and e', measured by tissue doppler. Using Vevo strain software analyzed and generated the left ventricular wall strain time curve, and records LS (longitudinal strain), RS (radial strain), CS (circumferential strain), LSR (longitudinal strain rate), RSR (radial strain rate) and CSR (circumferential strain rate).

Electrocardiograms: ECG (electrocardiograms) signals were collected with a multi-channel signal acquisition and processing system (RM6240BD, Chengdu, China) before injection and on the 7th and 14th day after injection. The ECG of mice in each group were collected by subcutaneously connecting the corresponding electrodes in the right upper limb, the right lower limb and the left upper limb. The software automatically analyzed the Q-wave amplitude, S-wave amplitude, T-wave amplitude, ST offset, PR interval, ST interval and average heart rate.

PET-CT : PET-CT was performed on the 7th and 14th day after injection. PET imaging in vivo was obtained by Siemens Inveon PET scanner. The mice were anesthetized with isoflurane, the induction concentration of inhaled isoflurane was $3 \%$, the maintenance concentration was $2 \%$, and ${ }^{18} \mathrm{~F}$-FDG $(120 \mu \mathrm{Ci} /$ mouse) was injected into tail vein. The mice were fixed on the scanning bed and kept still. The whole body of the mice was displayed in the field of vision. The data were collected in three-dimensional mode 20 minutes after the injection of ${ }^{18} \mathrm{~F}-\mathrm{FDG}$, manual location of mouse heart.

B-type natriuretic peptide: Before injection and the 7th and 14th day after injection, some mice were sacrificed and blood was taken. And then blood centrifugation, serum separation, storage backup. The BNP (B-type Natriuretic Peptide) in serum was calculated by mouse BNP ELISA (enzyme-linked immunosorbent assay) kit. The results were expressed in $\mathrm{pg} / \mathrm{ml}$.

Histomorphometry: On the 7th and 14th day after injection, the hearts of mice were sacrificed and the large blood vessels were removed and weighed. The cardiac tissues in different groups were prepared into frozen sections for histomorphometry analysis. Cardiac histology was examined by HE staining of thin myocardial sections. The pathological and morphological changes in the myocardial tissue were observed under an optical microscope.

Statistical analysis: The results of the experiment were analyzed by GraphPad Prism 5 software. All the data were expressed by Mean \pm SEM. T test was used for comparison between groups. When $P<0.05$, the difference was statistically significant.

\section{Results}

5-fluorouracil and bevacizumab reduced heart weight: According to the plan, the mice were randomly divided and male mice were used to exclude possible confounding factors caused by gender or hormone fluctuation. There was no statistical difference between the baseline body weight of each group. On the 7th and 14th day after injection, the body weight of the 5 -fluorouracil + bevacizumab group was lower than that of the control group, but there was no statistical difference $(P>0.05)$. As figure 1, part I. After 7 and 14 days of injection of 5 -fluorouracil and bevacizumab, the hearts of mice were washed and weighed, and the 
HWI (heart weight index) was calculated. On the 7th and 14th day, the heart weight index of 5- fluorouracil + bevacizumab group was obviously lower than that of the control group $(P<0.05)$. As figure 1 , part $\mathrm{J}$.

5-fluorouracil and bevacizumab increased ST offset and PR interval: On the 7th and 14th day after the injection of chemotherapy, the limb-lead ECG was recorded. The ST deviation increased on the 7 th and 14th day, but there was no statistical significance $(P>0.05)$. The P-R interval of 5 -fluorouracil + bevacizumab group was also longer on the 7 th and 14 th day, but there was no statistical significance $(P>0.05)$. There was no significant difference in $\mathrm{Q}$ wave between the control group and the 5 -fluorouracil + bevacizumab group on the 7th day after injection of the drug. On the 14th day, it was lower than the control group, but there was no statistical difference $(P>0.05)$. The $\mathrm{P}$-wave amplitude, $\mathrm{R}$-wave amplitude, T-wave amplitude, QRS interval, ST interval and QT interval of 5-fluorouracil + bevacizumab group had no significant difference compared with the control group $(P>0.05)$. The heart rate of 5 - fluorouracil + bevacizumab group was lower than that of the control group on the 7th and 14th day after injection, but there was no significant difference between the two groups $(P>0.05)$. As figure 1 .

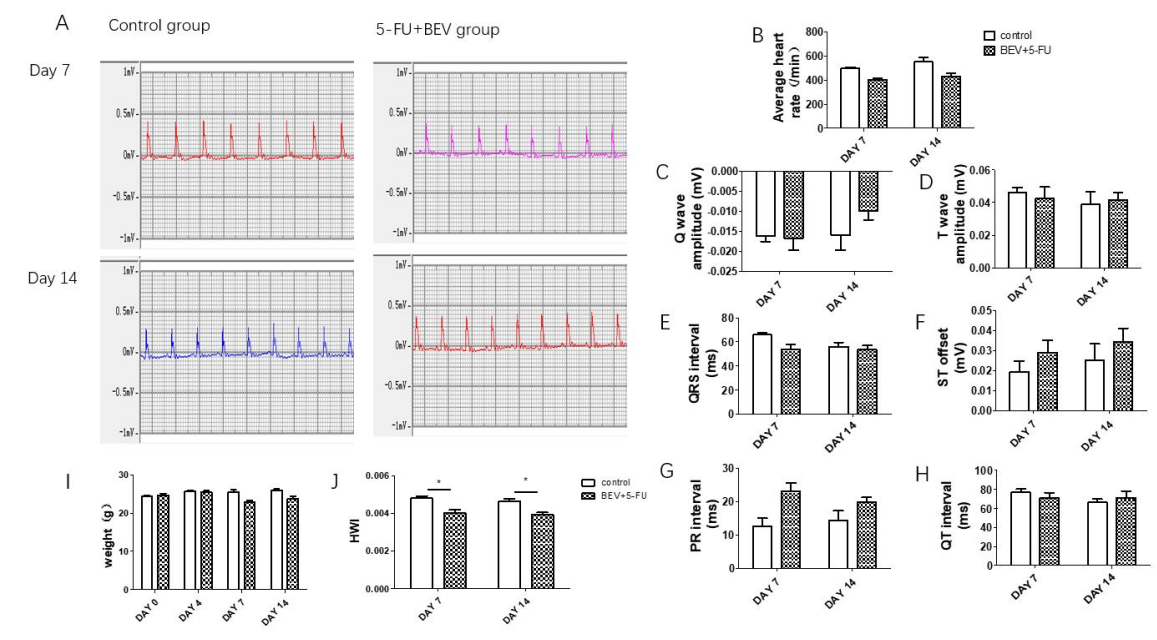

Figure 15 -fluorouracil and bevacizumab reduced heart weight and increased ST offset and PR interval. (n=8) A: Electrocardiogram showed small differences between control group and 5-fluorouracil + bevacizumab group. B: 5-fluorouracil + bevacizumab caused a small reduction in average heart rate. C: Q wave amplitude reduced on the 14th day after injection. D: No obvious changes in T wave amplitude were seen. E: No obvious changes in QRS interval were seen. F: ST offset increased in 5-fluorouracil + bevacizumab group on the 7th and 14th day after injection. G: PR interval increased in 5-fluorouracil + bevacizumab group on the 7th and 14th day after injection. H: No obvious changes in QT interval were seen. I: the body weight slightly reduced on the 7 th and 14 th day after injection. J: the heart weight index obviously reduced on the 7 th and 14th day after injection. 5-FU + BEV group: 5-fluorouracil + bevacizumab group.

The myocardial uptake increased on the 7 th day and decreased on the 14th day on PET-CT : In clinic, ${ }^{18} \mathrm{~F}$-FDG was used to detect myocardial metabolism and survival. Therefore, PET-CT was performed to the mice in each group on the 7th and 14th day after injection. On the 7th day after injection, the myocardial uptake of 5 -fluorouracil + bevacizumab group was significantly higher than that of the control group, and the cardiac uptake was diffusely increased, but the corresponding CT scan showed no significant changes in cardiac structure. On the 14 th day after injection, the ${ }^{18}$ F-FDG myocardial uptake in the 5fluorouracil + bevacizumab group was lower than that in the control group, and lower than that on the 7th day, even some of the ventricular walls were filling defect. And the defect walls were mainly the left ventricle inferior wall - posterior wall and posterior interval. At the same time, the corresponding CT scan showed no significant changes in the heart structure between the two groups. It can be seen that PET can detect cardiotoxicity caused by antineoplastic drugs in the early stage, and the increase of myocardial metabolism 
in the chemotherapy induced cardiotoxicity may be one of the early manifestations. As figure 3, part A.

No obvious changes in B-type natriuretic peptide concentration were seen: BNP (B-type natriuretic peptide) was a natural hormone with biological activity synthesized by cardiomyocytes, which was mainly expressed in ventricles and also existed in brain tissue. As a quantitative marker of heart failure, BNP not only reflects left ventricular systolic dysfunction, which also reflects left ventricular diastolic dysfunction, valve dysfunction and right ventricular dysfunction. Therefore, we measured the BNP content in serum of mice in each group. Compared with the control group, BNP in the serum of 5-fluorouracil + bevacizumab group on the 7 th and 14th day did not change significantly $(P>0.05)$, even in the serum of 5 -fluorouracil + bevacizumab group on the 14th day was slightly lower than that on the 7th day. As figure 3, part B.

Revealed myofibril irregular on the 14th day: After 7 and 14 days of injection of 5-fluorouracil and bevacizumab, the hearts of mice were washed and weighed. After HE staining of myocardial sections, it was observed that some of the myofibril arranged irregularly in a small range after 5 -fluorouracil and bevacizumab were given. As figure 3, part C.

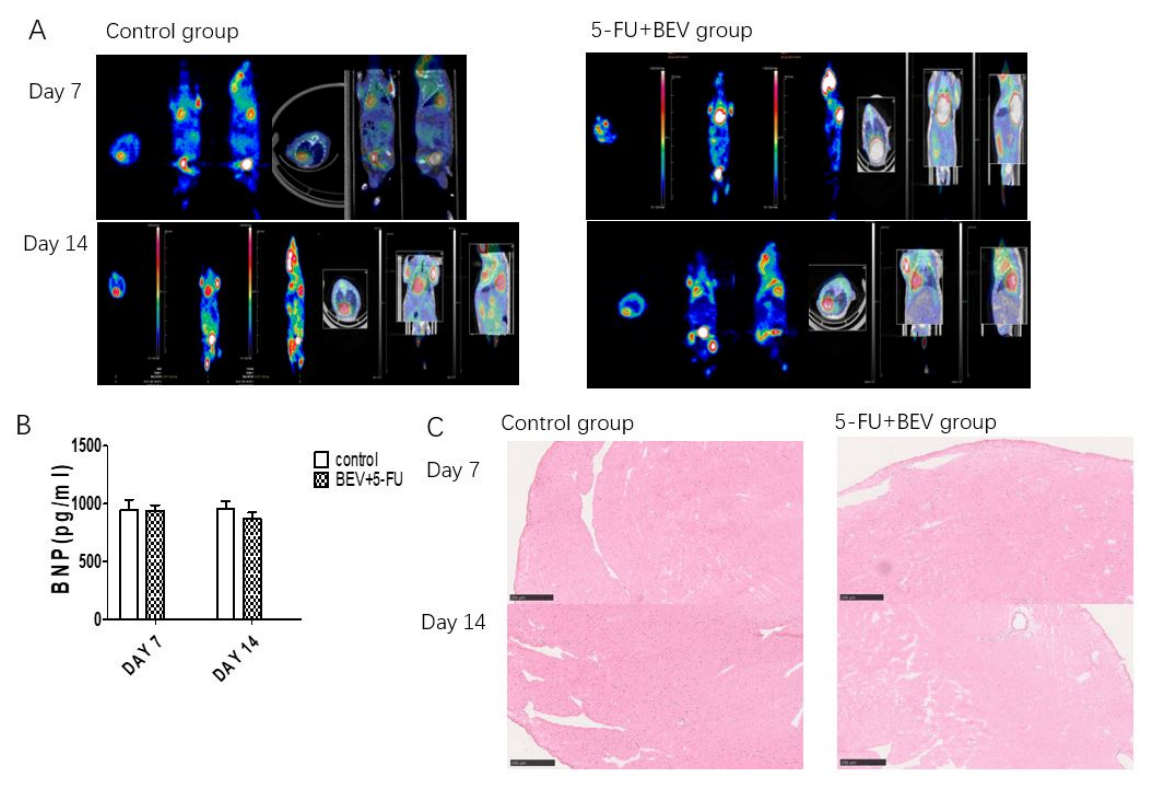

Figure 2 PET-CT images, BNP, HWI and HE staining of myocardial sections of each group on the 7th and 14th day after injection. A: PET-CT images showed high uptake on the 7th and low uptake on the 14th day. $(\mathrm{n}=6)$ B: No obvious changes in BNP were seen. $(\mathrm{n}=8)$ C: HE staining of myocardial sections showed some myocardial cell destruction in 5 -fluorouracil + bevacizumab group on the 14th day after injection (objective $\times 10)$. 5-FU + BEV group: 5-fluorouracil + bevacizumab group.

LVPW; d decreased on the 7th and 14th day and IVS;d, EF, FS decreased on the 14th day: As a convenient method to detect the function and structure of the heart, echocardiography was performed before injection and on the 4th, 7 th and 14th days after injection to evaluate the changes of left ventricular function and structure in mice. Before and the 4th, 7th and 14th days after injection, there was no obvious cardiac echo change in the echocardiogram of mice in each group. The mitral valve, tricuspid valve and aortic valves were soft and well opened, and there was no obvious reflux signal. There was no obvious change in the heart structure of mice in each group. Compared with the control group, the IVS;d and LVPW;d in the 5-fluorouracil + bevacizumab group became thinner with the progress of the disease, and there was a statistical difference $(P<0.05)$ on the 14th day compared with the control group. The LVID;d and LVID;s slightly increased, but no statistical difference $(P>0.05)$. LVEF and LVFS decreased significantly on the 7 th and 14th day compared with the control group. This indicated that 5-fluorouracil and bevacizumab had 
certain damage to the heart and had a certain correlation with the dosage. As figure 3, part A-G.

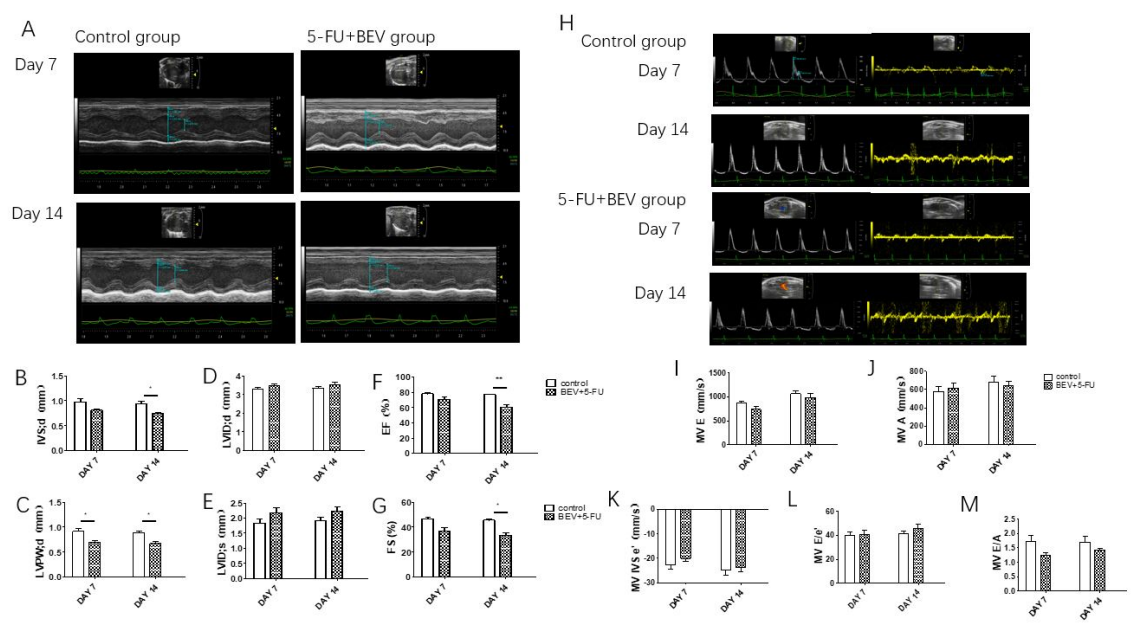

Figure 3 LVPW; decreased on the 7th and 14th day and IVS;d, EF, FS decreased on the 14th day after injection in the 5-fluorouracil + bevacizumab group. $(\mathrm{n}=10) \mathrm{A}$ : M mode of each group on the 7 th day and 14th day. B: IVS;d obviously decreased on the 14th day. C: LVPW;d decreased obviously on the 7th and 14th day. D: LVID;d slightly increased on the 7th and 14th day. E: LVID;s slightly increased on the 7th and 14th day. F: EF obviously decreased on the 14th day. G: FS obviously decreased on the 14th day. H: Spectral doppler and tissue doppler imaging of each group on the 7th day and 14 th day. I: No obvious changes in MVE were seen. J: No obvious changes in MVA were seen. K: No obvious changes in MV IVS e' were seen. L: MV E/A of 5-fluorouracil + bevacizumab group decreased on the 7th and 14th day. M: MV E/e' slightly increased on the 14th day after injection. 5-FU + BEV group: 5-fluorouracil + bevacizumab group. *: $\mathrm{P}_{\mathrm{j}} 0.05 .{ }^{* *}: \mathrm{P}_{\mathrm{j}} 0.01$.

There were no obvious changes in MV E, MV A and MV IVS e' of 5-fluorouracil+ bevacizumab group were seen, while MV E/e' slightly increased on the 14th day after injection. MV E/A of 5-fluorouracil + bevacizumab group decreased on the 7 th and 14th day, but there was no significant difference $(P>0.05)$. Therefore, we can observe that 5-fluorouracil and bevacizumab had little effect on diastolic function, and they were difficult to detect in conventional echocardiography. As figure 3, part H-M.

Global longitudinal strain decreased at first on the 7th day: In order to verify the application of speckle tracking echocardiography in the diagnosis of chemotherapy induced cardiotoxicity, we used VEVO strain to analyze the left ventricular wall motion of mice in each group. The GLS (global longitudinal strain) of left ventricular in 5-fluorouracil + bevacizumab group was significantly lower than that in the control group $(P<0.05)$ on the 7 th and 14th day. The long axis GRS (global radial strain) was lower than that in the control group $(P<0.05)$ on the 14 th day. And the GCS (global circumferential strain) was significantly lower than that in the control group $(P<0.05)$ on the 14 th day. On the 7 th and 14 th day, the short axis GRS was lower than that of the control group, but there was no statistical difference $(P>0.05)$. While the long axis GLSR (global longitudinal strain rate) and the long axis GRSR (global radial strain rate) were only slightly lower than that of the control group on the 14th day, but there was no statistical difference $(P$ $>0.05$ ). The short axis GCSR (global circumferential strain rate) and the short axis GRSR were lower than that of the control group on the 7 th and 14 th day, but there was no statistical difference $(P>0.05)$. As figure 4 . 

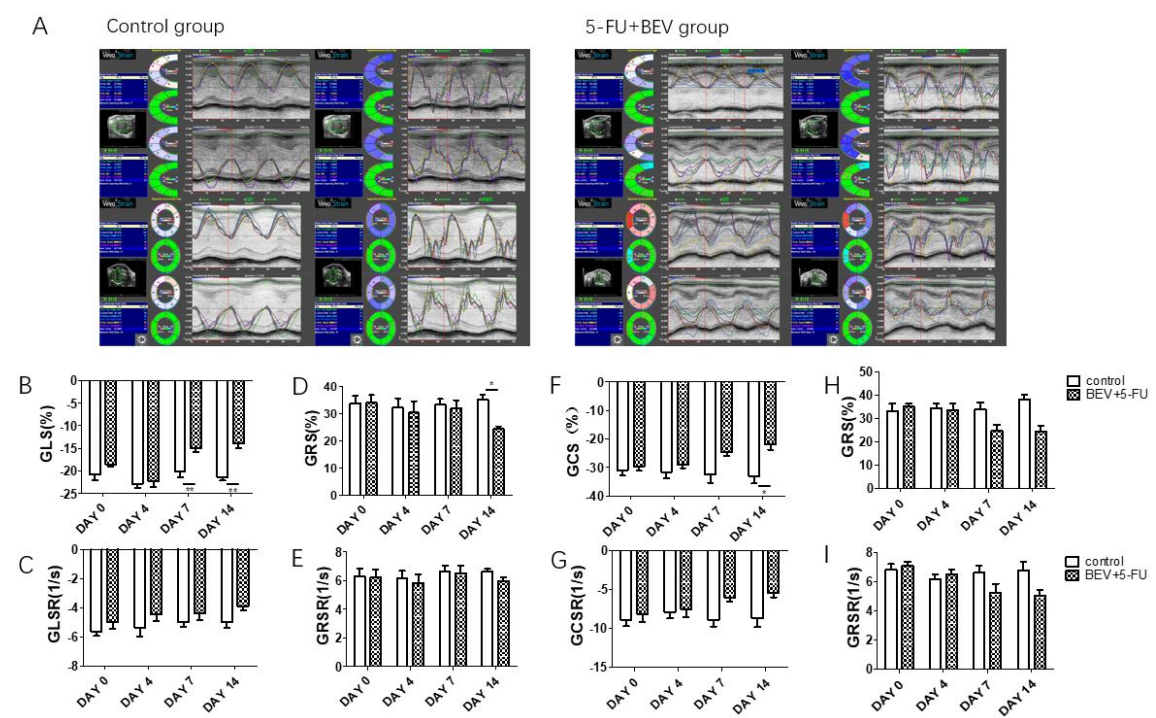

Figure 4 Global strain of left ventricle long axis and short axis obviously decreased and global strain rate of left ventricle long axis and short axis slightly decreased in 5-fluorouracil + bevacizumab group. ( $\mathrm{n}=10)$ A: Long axis and short axis strain and strain rate of each segment of left ventricle of each group. B: GLS firstly decreased in 5-fluorouracil + bevacizumab group on the 7th day. C: GLSR slightly decreased in 5-fluorouracil + bevacizumab group with time. D: GRS of long axis obviously decreased in 5-fluorouracil + bevacizumab group the 14th day. E: GRSR of long axis slightly decreased in 5-fluorouracil + bevacizumab group with time. F: GCS obviously decreased in 5-fluorouracil + bevacizumab group the 14th day. G: GCSR of long axis slightly decreased in 5-fluorouracil + bevacizumab group with time. H: GRS of short axis decreased in 5-fluorouracil + bevacizumab group with time. I: GRSR of short axis slightly decreased in 5-fluorouracil + bevacizumab group with time. 5-FU + BEV group: 5 -fluorouracil + bevacizumab group. ${ }^{*}: P\left\lceil 0.05\right.$. ${ }^{* *}: P$ $¡ 0.01$.

The analysis of the strain and strain rate of each segment of left ventricle: It can be seen that the long axis radial strain of the posterior wall decreased on the 7th day, while the radial strain of the anterior wall of the left ventricle decreased on the 14th day. In the long axis longitudinal strain, the strain of the apical segment decreased on the 7th day compared with the control group, while the strain of the middle and basal segments decreased on the 14th day compared with the control group potential. The short axis radial strain in 5-fluorouracil + bevacizumab group was significantly lower than that in the control group on the 7th and 14th day, among which the left ventricular anterior wall, lateral wall, posterior wall and inferior wall had statistical difference $(P<0.05)$, but there was no statistical difference in the interventricular septum $(P>0.05)$. The short axis circumferential strain of left ventricular anterior wall, anterior wall, posterior wall and inferior wall was significantly lower on the 7th and 14th day than that in the control group. And there was significant difference in the anterior wall of left ventricle $(P<0.05)$. As figure 5 and 6 . 

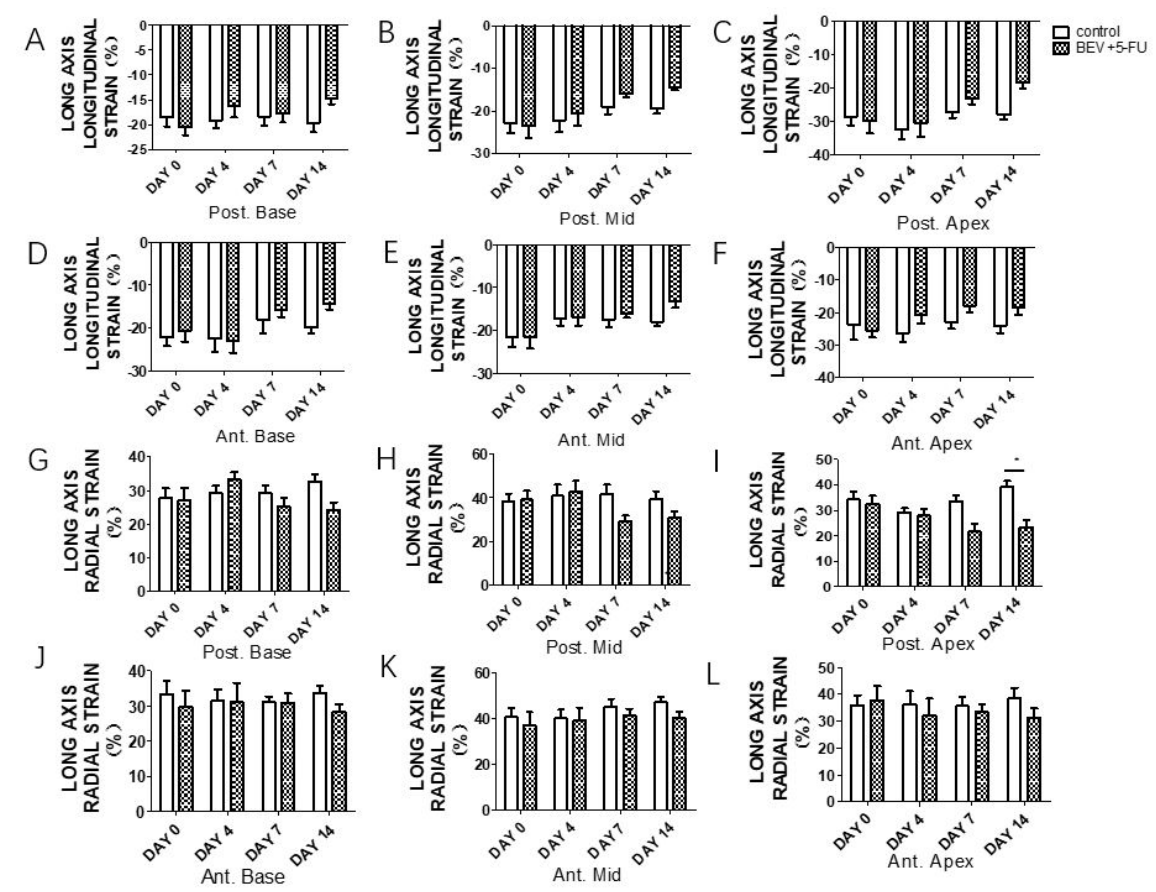

Figure 5 The decrease of long axis radial strain firstly appeared in the posterior wall, while the decrease of the long axis longitudinal strain firstly appeared in the apical segment in the 5 -fluorouracil + bevacizumab group. $(\mathrm{n}=10) \mathrm{A}$ : The long axis longitudinal strain of poet. base. B: The long axis longitudinal strain of poet. mild. C: The long axis longitudinal strain of poet. apex. D: The long axis longitudinal strain of ant. base. E: The long axis longitudinal strain of ant. mild. F: The long axis longitudinal strain of ant. apex. G: The long axis radial strain of poet. base. H: The long axis radial strain of poet. mild. I: The long axis radial strain of poet. apex. J: The long axis radial strain of ant. base. K: The long axis radial strain of poet. apex. L: The long axis radial strain of poet. apex. 5-FU + BEV group: 5-fluorouracil + bevacizumab group. *: $P$ ¡0.05. 

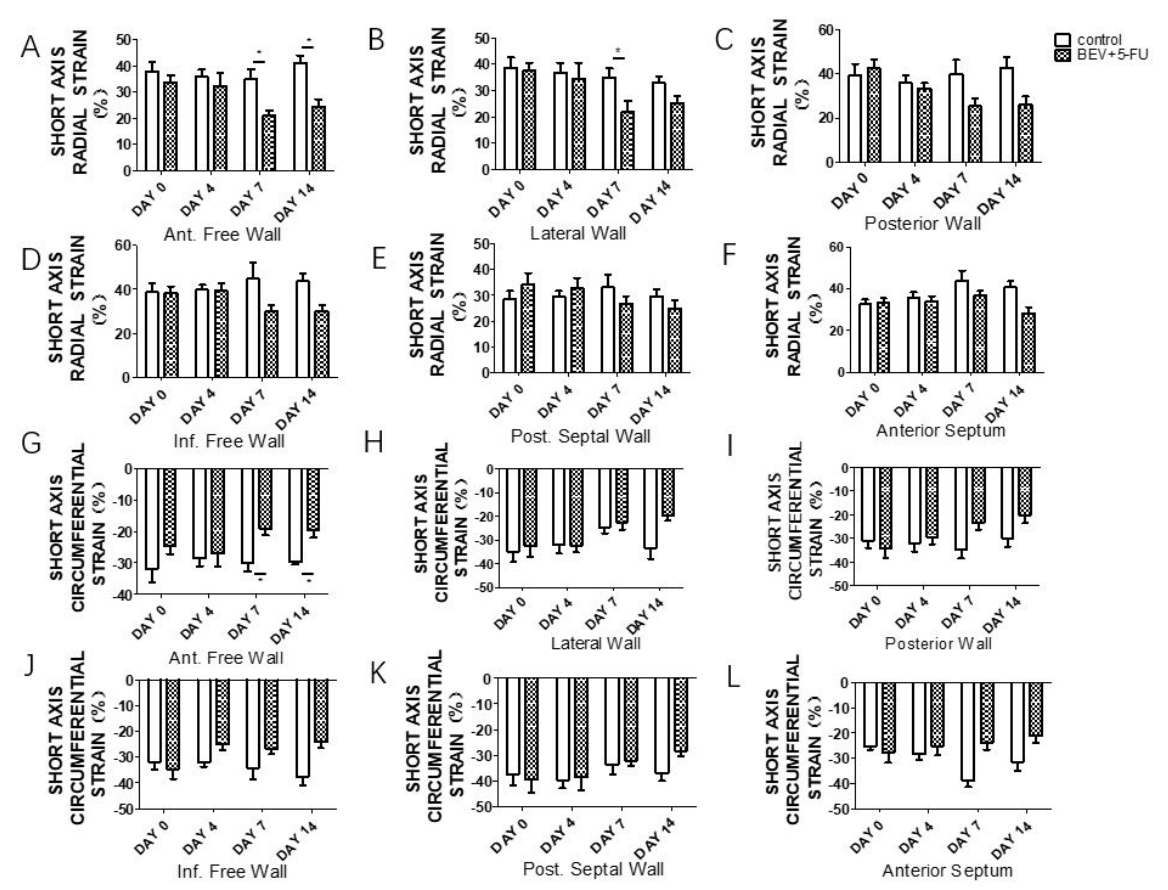

Figure 6 The decrease of the short axis radial strain firstly appeared in the left ventricular anterior wall, lateral wall and posterior wall, while the decrease of the short axis circumferential strain firstly appeared in the left ventricular anterior wall. $(\mathrm{n}=10)$ A: The short axis radial strain of ant. free wall. B: The short axis radial strain of lateral wall. C: The short axis radial strain of posterior wall. D: The short axis radial strain of inf. free wall. E: The short axis radial strain of post. septal wall. F: The short axis radial strain of anterior septum. G: The short axis circumferential strain of ant. free wall. H: The short axis circumferential strain of lateral wall. I: The short axis circumferential strain of posterior wall. J: The short axis circumferential strain of ant. base. K: The short axis circumferential strain of post. septal wall. L: The short axis circumferential strain of anterior septum. 5-FU + BEV group: 5-fluorouracil + bevacizumab group. *: $P$ ¡0.05.

In the aspect of strain rate, the long axis radial strain rate of each segment of left ventricle was significantly different on the 7th day, slightly lower than that of the control group on the 14th day. The long axis longitudinal strain rate of left ventricle was slightly lower than that of the control group on the 14th day, in which the decrease of left ventricular apical segment was larger than that of the middle segment and basal segment, but there was no statistical difference $(P>0.05)$. The short axis radial strain rate of left ventricular anterior wall, posterior wall, inferior wall and anterior interval was lower than that of the control group on the 14th day, but there was no significant difference in the left ventricular lateral wall and posterior septum. The short axis circumferential strain rate of each segment of the left ventricle decreased in different degrees on the 7 th and 14th day, but there was no statistical difference $(P>0.05)$. As figure 7 and 8 . 


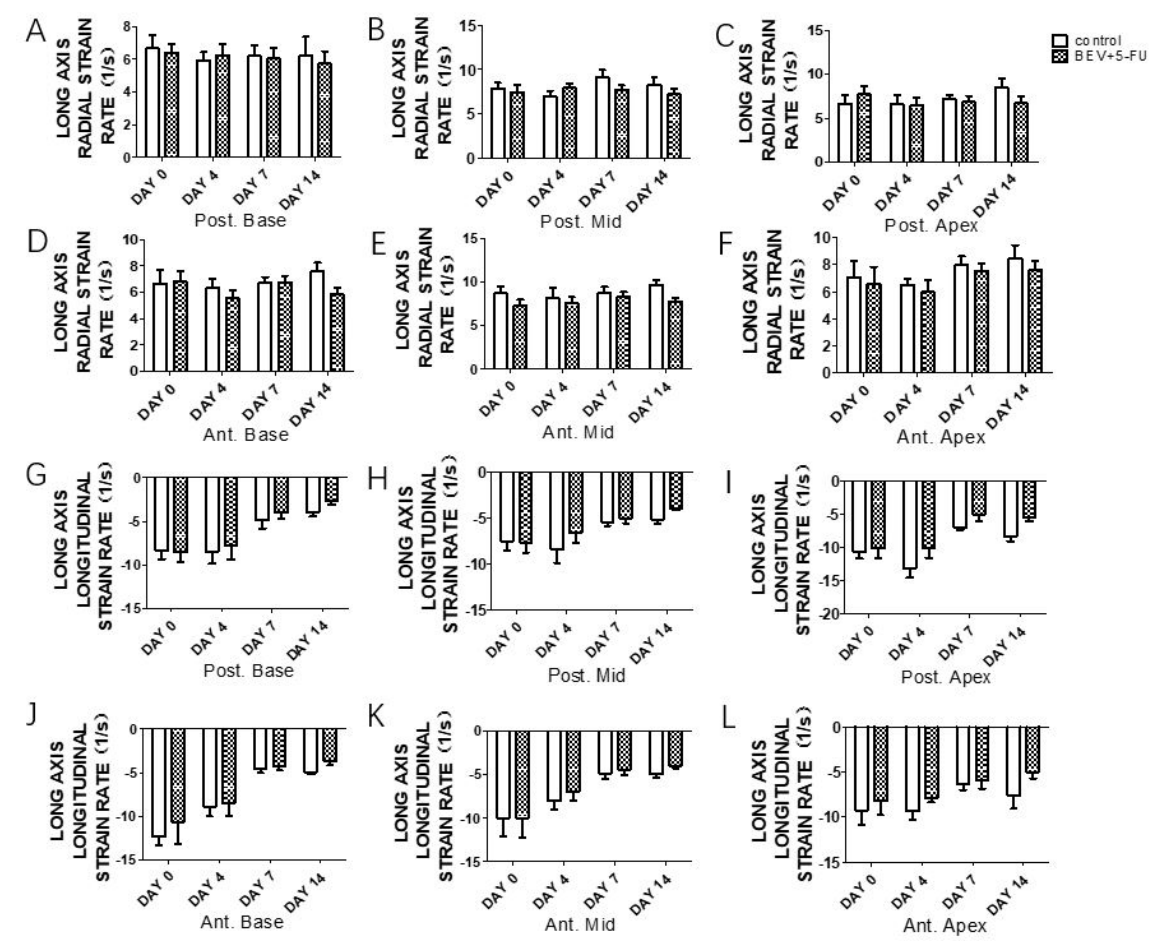

Figure 7 Strain rate of the long axis slightly decreased of left ventricular segments. $(n=10)$ A: The long axis radial strain rate of poet. base. B: The long axis radial strain rate of poet. mild. C: The long axis radial strain rate of poet. apex. D: The long axis radial strain rate of ant. base. E: The long axis radial strain rate of poet. apex. F: The long axis radial strain rate of poet. apex. G: The long axis longitudinal strain rate of poet. mild. H: The long axis longitudinal strain rate of poet. base. I: The long axis longitudinal strain rate of poet. apex. J: The long axis longitudinal strain rate of ant. base. K: The long axis longitudinal strain rate of ant. mild. L: The long axis longitudinal strain rate of ant. apex. 5-FU + BEV group: 5-fluorouracil + bevacizumab group. 


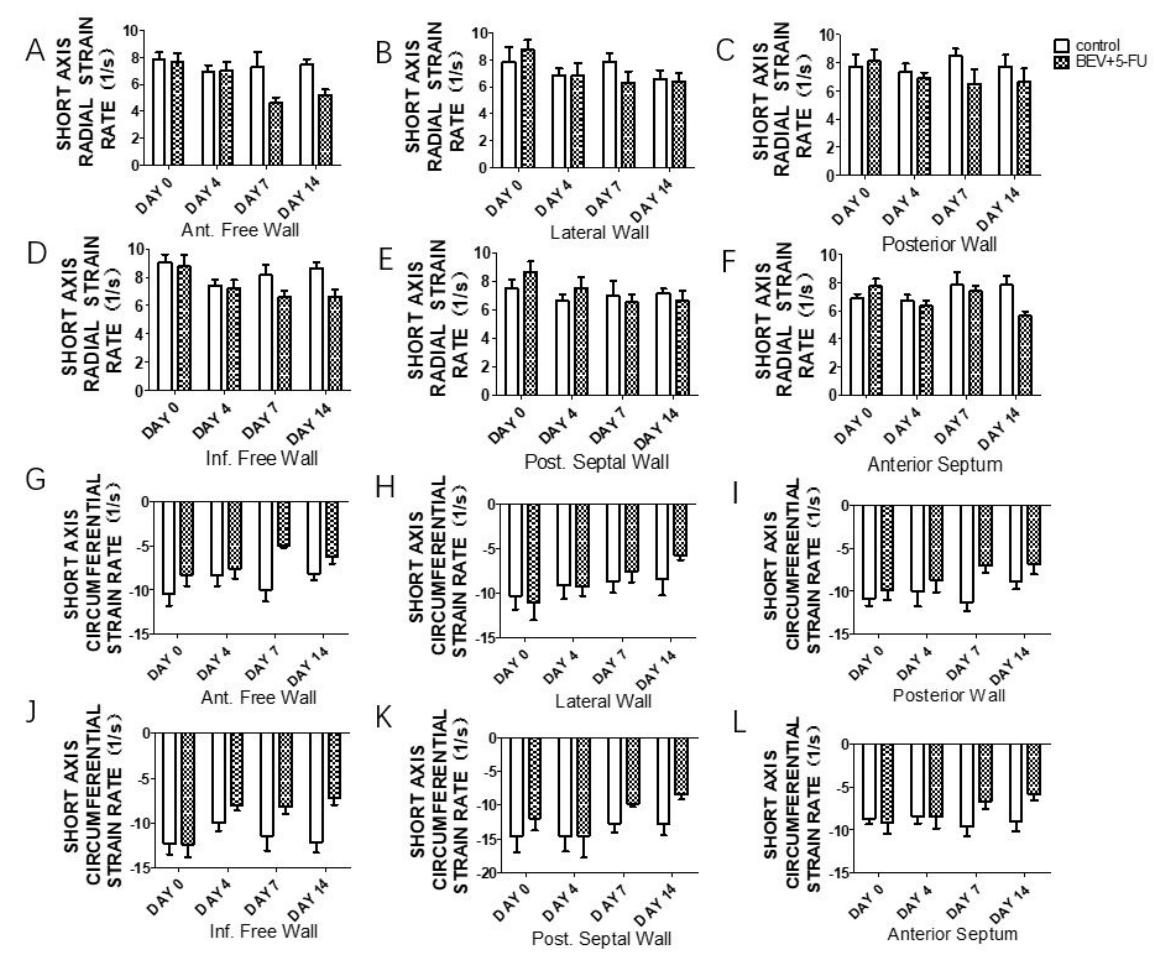

Figure 8 The decrease of the short axis radial strain rate firstly appeared in the left ventricular anterior wall, posterior wall and inferior wall, while the short axis circumferential strain rate showed different decrease in each segment. $(n=10)$ A: The short axis radial strain rate of ant. free wall. B: The short axis radial strain rate of lateral wall. C: The short axis radial strain rate of posterior wall. D: The short axis radial strain rate of inf. free wall. E: The short axis radial strain rate of post. septal wall. F: The short axis radial strain rate of anterior septum. G: The short axis circumferential strain rate of ant. free wall. H: The short axis circumferential strain rate of lateral wall. I: The short axis circumferential strain rate of posterior wall. $\mathrm{J}$ : The short axis circumferential strain rate of ant. base. K: The short axis circumferential strain rate of post. septal wall. L: The short axis circumferential strain rate of anterior septum. 5-FU + BEV group: 5-fluorouracil + bevacizumab group.

\section{Discussion}

Since 1957, 5 -fluorouracil has become one of the first members of fluorouracil chemotherapy. Soon it become one of the most widely used drugs for solid tumors, and has become the basic drug of FOLFOX chemotherapy program. Then bevacizumab has improved the anti-tumor program, and the effect of chemotherapy was more significant. But at the same time, the use of them was not without risk. At present, the reported incidence of cardiotoxicity caused by the use of 5-fluorouracil and bevacizumab varies, and its damage mechanism was also diverse. However, it was crucial to detect and diagnose the anti-tumor drug cardiotoxicity in the early stage and adjust the chemotherapy program, so as to prevent unnecessary morbidity and mortality. According to the current definition of cardiotoxicity caused by antitumor drugs, LVEF $550 \%$ or LVEF reduced by $10 \%$ compared with baseline are clinical cardiotoxicity symptoms ${ }^{[6 】}$. In this study, different detection technologies were explored to early diagnosis cardiotoxicity caused by antitumor drugs.

ECG, as the most basic and convenient technology for clinical detection of cardiac function, has been widely used. In the detection of chemotherapy induced cardiotoxicity, we showed a decrease in heart rate and an extension of PR interval on the 7th day, suggesting that 5-fluorouracil and bevacizumab may affect the cardiac electrical conduction system, hinder the conduction of electrocardiograph. And ST segment elevation suggested that 5-fluorouracil and bevacizumab may injure system of coronary circulation. However, there 
was no significant difference in ECG between the two groups, which indicated that the sensitivity of ECG in the detection of chemotherapy induced cardiotoxicity was low. At the same time, many other heart diseases that affect the coronary artery can also show similar ECG performance, so it needs to be further evaluated with other detection methods ${ }^{[7]}$.

In this study, the BNP of 5-fluorouracil + bevacizumab group mice had no significant change at all time points, probably because the heart injury was not very serious. But some literatures pointed out that the BNP was normal or not increased in the heart injury, indicating the poor prognosis. At the same time, the change of serum BNP content appeared the time varies from 1 month to 12 months. And if there was no other index support, BNP elevation alone can not diagnose cardiac insufficiency [8】. As for myocardial histopathology, compared with other detection techniques, although it was the gold standard for the diagnosis of myocardial condition [9], the heart function of patients may have been greatly reduced before the heart pathology changing. In this study, the degree of mouse heart was less damaged, it was difficult for conventional myocardial histopathology to change in a large range. And it was not suitable used for early detection of cardiotoxicity because of invasive.

The ${ }^{18}$ F-FDG mediated PET myocardial imaging can directly show the metabolism of mouse heart. We showed the increase of myocardial uptake on the 7th day after injection, suggesting that it may be an early manifestation of chemotherapy induced cardiotoxicity. This may be related to the decrease of mitochondrial oxidation and the abnormal metabolism of glycolysis energy produced by increased lactic acid, such metabolic recombination may cause the activity of hexokinase the increase of ${ }^{18} \mathrm{~F}$-FDG phosphorylation lead to the

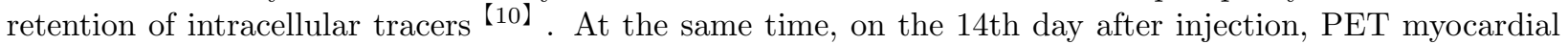
imaging can detect myocardial damage more sensitively than other detection techniques, especially the damage of left ventricle inferior wall - posterior wall and posterior interval. But compared with other detection techniques, PET was more complicated and had radiation to human body. Some studies have shown that the changes of myocardial glucose metabolism can be detected early in the course of anthracycline treatment. Through the detection of FDG intake, the cardiac glucose consumption gradually increases or even reached a higher level during and after chemotherapy, and the incidence of cardiac toxicity also increases 【11】. However, CT shows its disadvantage in the detection of chemotherapy induced cardiotoxicity. Because most of cardiotoxicity caused by antineoplastic drugs has functional changes first, and structural changes occur later, the incidence is also low, so CT can not detect cardiotoxicity in the early stage.

Conventional echocardiography, as an imaging method for cardiac detection, has been recommended by oncology and cardiology guidelines as the main screening technology for cardiotoxicity of antineoplastic drugs because of its advantages such as low cost, wide application, no ionizing radiation and high patient acceptance. It can not only judge heart failure and left ventricular systolic dysfunction, but also observe structural changes in the heart caused by cancer treatment. In this study, the IVS, LVPW thickness, LVEF and LVFS gradually decreased, and there were statistical differences on the 14th day. In addition, we also evaluated left ventricular diastolic function. The MVA, MVE, e' and E/e' did not change significantly within 14 days after injection. Only MVE/A decreased on the 7th day, but there was no statistical difference. Therefore, 5-fluorouracil and bevacizumab had little effect on left ventricular diastolic function. So we showed that left ventricular diastolic function cannot be used for early detection of cardiotoxicity induced by 5 -fluorouracil and bevacizumab. However, it has been reported that the left ventricular diastolic function can change in the early stage of predicting adriamycin induced cardiotoxicity ${ }^{[12\rfloor}$, which may be related to the injury mechanism of antitumor drugs.

What more, we confirmed that the decrease of speckle tracking echocardiography was earlier than that of conventional echocardiography, which could be a sign of the subsequent decrease of cardiac function. Speckle tracking echocardiography was a good method to measure myocardial movement, in which strain reflected the degree of myocardial motion in a cardiac cycle, and strain rate was the rate of myocardial systole and diastole. The advantage of strain and strain rate measurement was that it can distinguish the movement of each myocardial segment and analyze the abnormal myocardial movement irrelevant to normal myocardial

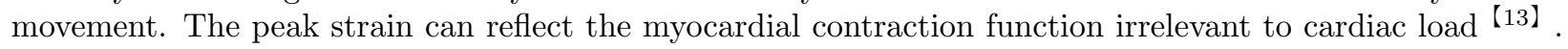


Therefore, the measurement of strain and strain rate can provide the heart's own collection Information about the shrink function. A number of studies have indicated that GLS can detect left ventricular dysfunction before LVEF decreases, and it is one of the independent predictors of mortality in all cardiotoxicity [14】. Currently, the guideline recommends $-15 \%$ as subclinical indicators of left ventricular dysfunction [15】.

Compared with strain and strain rate, we found that the former can detect the heart injury earlier. Among them, GLS first changed in overall strain. On the 7th day after injection, when LVEF had not changed significantly, GLS decreased with statistical difference, while GRS and GCS decreased later than GLS. It has been reported that the decrease of GLS may be related to the dose of chemotherapy or radiotherapy [16】. However, there was no clear research on why GLS was the earliest indicator of overall strain change.

In addition to focusing on the global strain, we found that the strain of different segments of the left ventricle changed at different times. The decrease of the longitudinal strain of the long axis first occurred in the left ventricle apical segment. In the aspect of left ventricular long axis radial strain, the posterior wall decreased earlier than the interventricular septum. In the observation of left ventricular short axis strain, we found that the anterior wall of left ventricle decreased first in both short axis radial strain and circumferential strain. It can be seen that the area dominated by the anterior descending branch of the left coronary artery may be more sensitive to the decrease of the cardiac function in the strain index of the left ventricular short axis.

In conclusion, we showed that compared with other cardiac detection techniques, speckle tracking echocardiography and ${ }^{18}$ F-FDG myocardial imaging can detect myocardial damage in early stage, but speckle tracking echocardiography is more convenient. And GLS is the best indicator of cardiotoxicity in spot tracking echocardiography. The decrease of the longitudinal strain of the long axis of the left ventricle first occurred in the apical segment of the left ventricle, the decrease of the radial strain of the long axis of the left ventricle first occurred in the posterior wall of the left ventricle, and the decrease of the radial strain and the circumferential strain of the short axis of the left ventricle first occurred in the anterior wall of the left ventricle.

In this study, we discussed the early detection of chemotherapy induced cardiotoxicity by a variety of detection techniques. The chemotherapy induced cardiotoxicity has a great influence on the expected survival rate of patients. To control the adverse reactions of anti-tumor drugs was important in clinical medication. Therefore, the establishment of more effective early diagnosis methods to reduce or minimize chemotherapy induced cardiotoxicity was desired. Fully understanding and timely monitoring the cardiotoxicity of drugs was beneficial to the prognosis of patients. In this way, we can provide the best treatment plan for patients, and make anti-tumor drugs safer and more effective for the benefit of cancer patients.

\section{Reference}

【1】 Bray Freddie, Ferlay Jacques, Soerjomataram Isabelle, , et al . Global cancer statistics 2018: GLOBOCAN estimates of incidence and mortality worldwide for 36 cancers in 185 countries. $[\mathrm{J}]$. CA: a cancer journal for clinicians, 2018;1:1-31.

【2】 Siegel Rebecca L,Miller Kimberly D,Jemal Ahmedin. Cancer statistics, 2015.[J]. CA: a cancer journal for clinicians, 2015,65(1).

【3】 Wanqing Chen, Rongshou Zheng, Siwei Zhang, ,et al . Cancer incidence and mortality in China in 2013:an analysis based on urbanization level[J].Chinese Journal of Cancer Research,2017,29(1):1-10.

【4】 Savage P,Mahmoud S. Development and economic trends in cancer therapeutic drugs: a 5-year update 2010-2014.[J]. British journal of cancer,2015,112(6).

【5】 National Comprehensive Cancer Network. NCCN clinical practice guidelines in Oncology:colorectal cancer screening (2018.V1)[EB/OL].2018-3-26

【6】 Gian Marco Rosa, Lorenzo Gigli, Maria Isabella Tagliasacchi, et al . Update on cardiotoxicity of anticancer treatments[J]. European Journal of Clinical Investigation,2016,46(3). 
【7】 Allison John D,Tanavin Toug, Yang Yang,Birnbaum Gilad,Khalid Umair. Various Manifestations of 5-Fluorouracil Cardiotoxicity: A Multicenter Case Series and Review of Literature.[J]. Cardiovascular toxicology,2020.

【8】Zhang Cheng,Shi Dan,Yang Ping. BNP as a potential biomarker for cardiac damage of breast cancer after radiotherapy: a meta-analysis.[J]. Medicine,2019,98(29).

【9】Jun Ma, Zhixiang Shen, Shukui Qin. Consensus on the prevention and treatment of cardiotoxicity of anthracycline antitumor drugs among Chinese experts (2011). [J].Chinese Clinical Oncology, 2011,16(12):11221129 .

【10】 Sarocchi Matteo, Bauckneht Matteo, Arboscello Eleonora, et al . An increase in myocardial 18fluorodeoxyglucose uptake is associated with left ventricular ejection fraction decline in Hodgkin lymphoma patients treated with anthracycline.[J]. Journal of translational medicine,2018,16(1).

【11】 Bauckneht Matteo, Ferrarazzo Giulia, Fiz Francesco, et al . Doxorubicin Effect on Myocardial Metabolism as a Prerequisite for Subsequent Development of Cardiac Toxicity: A Translational 18FFDG PET/CT Observation.[J]. Journal of nuclear medicine : official publication, Society of Nuclear Medicine,2017,58(10).

【12】 Nagiub Mohamed,Nixon John V,Kontos Michael C. Ability of Nonstrain Diastolic Parameters to Predict Doxorubicin-Induced Cardiomyopathy: A Systematic Review With Meta-Analysis.[J]. Cardiology in review,2018,26(1).

【13】Thavendiranathan Paaladinesh,Poulin Frédéric,Lim Ki-Dong,Plana Juan Carlos, Woo Anna,Marwick Thomas H. Use of myocardial strain imaging by echocardiography for the early detection of cardiotoxicity in patients during and after cancer chemotherapy: a systematic review.[J]. Journal of the American College of Cardiology,2014,63(25 Pt A).

【14】Romano Simone, Judd Robert M, Kim Raymond J, et al . Association of Feature-Tracking Cardiac Magnetic Resonance Imaging Left Ventricular Global Longitudinal Strain With All-Cause Mortality in Patients With Reduced Left Ventricular Ejection Fraction.[J]. Circulation,2017,135(23).

【15】Zamorano Jose Luis, Lancellotti Patrizio, Rodriguez Muñoz Daniel,et al . 2016 ESC Position Paper on cancer treatments and cardiovascular toxicity developed under the auspices of the ESC Committee for Practice Guidelines: The Task Force for cancer treatments and cardiovascular toxicity of the European Society of Cardiology (ESC).[J]. European heart journal,2016,37(36).

【16】 Walker Valentin, Lairez Olivier, Fondard Olivier, et al . Early detection of subclinical left ventricular dysfunction after breast cancer radiation therapy using speckle-tracking echocardiography: association between cardiac exposure and longitudinal strain reduction (BACCARAT study). [J]. Radiation oncology (London, England),2019,14(1).

\section{Hosted file}

Figure.docx available at https://authorea.com/users/329838/articles/456724-early-assessmentof-5-fluorouracil-and-bevacizumab-induced-cardiotoxicity-by-multimodal-imagingtechniques 